\title{
Constructions of Robust Protocol Sequences for Wireless Sensor and Ad hoc Networks
}

\author{
Chung Shue Chen, Member, IEEE, Wing Shing Wong, Fellow, IEEE, and Ye-Qiong Song
}

\begin{abstract}
A class of periodic unipolar binary sequences is investigated for their potential applications in defining new protocols for distributed multiple accessing. Based on linear congruence sequences, one can show that, for any finite subset of these sequences, with the total proportional rate not exceeding a specific threshold, there cannot be enough collisions to completely block any particular sequence, no matter how they are shifted with respect to one another. This property can be exploited in certain applications, such as wireless sensor and ad hoc networks. A further investigation into how to enhance the allowable rate sum is conducted. New protocol sequences with interesting and useful properties are accordingly designed.
\end{abstract}

Index Terms-Collision channel without feedback, multiple access, protocol sequence, shift invariant, user unsuppressibility.

\section{INTRODUCTION}

D IGITAL sequences have found many civilian and military applications over the past semicentury. Pseudonoise (PN) sequence [1] is one of the well-known examples. Typically, they can be classified into binary and nonbinary sequences with broad usage in various signal processing and communication systems. Applications may require specific correlation properties and structures [2]. For example, in spread-spectrum systems, cross correlation between spreading codes has a significant impact on the system performance since it reflects mutual interference among users. The number of sequences available is also one of the key measures in identifying the importance of a sequence set. Usually, there are tradeoffs between these properties due to different applications.

In wireless sensor networks [3]-[5], due to computing power limitations and strict energy-consumption constraints, it will be favorable to have a simple multiple-access protocol that requires neither frequent monitoring of the channel for feed-

Manuscript received December 2, 2006; revised June 18, 2007 and October 24, 2007. This work was supported by the Research Grants Council of the Hong Kong Special Administrative Region under Project 416906. This paper was presented in part at IEEE Globecom'07, Washington, DC, November 26-30, 2007. The review of this paper was coordinated by Prof. J. Li.

C. S. Chen was with Lorraine Laboratory of IT Research and Applications (LORIA)-Centre National de la Recherche Scientifique, 54600 Villers Les Nancy, France. He is now with the Department of Electronics and Telecommunications, Norwegian University of Science and Technology, 7491 Trondheim, Norway (e-mail: cschen@iet.ntnu.no).

W. S. Wong is with the Department of Information Engineering, The Chinese University of Hong Kong, Shatin, Hong Kong (e-mail: wswong@ie. cuhk.edu.hk).

Y.-Q. Song is with LORIA-Institut National Polytechnique de Lorraine, 54506 Vandoeuvre-les-Nancy, France (e-mail: song@loria.fr).

Digital Object Identifier 10.1109/TVT.2007.914478 back information nor complicated processing, such as a backoff algorithm and random number generation. In addition, when considering an ad hoc system with dynamic network topology [6], which may be due to user mobility or time-varying propagation delays, sharing a radio channel among a large number of devices with the requirement of well-coordinated transmissions and time offsets could be very complicated. This is particularly hard for thin devices. The preceding reasons lead to our succeeding explorations.

To have a simple multiple-access scheme for wireless sensor and ad hoc networks, the idea of using deterministic coding sequences to define random accessing in collision channel without feedback [7] is worth a revisit and consideration. It is desirable to avoid the need for feedbacks and retransmissions, particularly in a mobile environment [8]. Unipolar periodic binary sequences are employed as protocol sequences [9] to address when a user can transmit and when it should be silent. Some related works and discussions can be found in [10]-[23] In [10], linear congruence sequences were designed for timefrequency hopping systems. Prime sequences were constructed [11] and applied in spread-spectrum optical networks [12]. They are closely related to the linear congruence sequences but originally constructed for different purposes [13]. In [14], some concatenated prime sequences are used for collision resolution in contention access local area networks, and the result has shown their superiority over some backoff algorithms. The use of constant-weight cyclically permutable codes for multipleaccess collision channels is reported in [15] and [16]. The performance of these sequences relies on the linear cyclic codes employed.

In [17], a new family of protocol sequences built on the concept of prime sequences, namely wobbling sequences, is designed to support multirate communication and service guarantee in random-access channels. In general, the maximum number of allowable users, their throughput performance, and the length of sequence period are the major concerns. Meanwhile, correlation properties between sequences are often the important indicators [18]. For practical considerations, coding across packets can be applied to recover data lost or correct erasures due to possible collisions, following typical algorithms in the literature [19]-[21]. Discussions on some advanced coding and decoding schemes can also be found in [8] and [22], in which techniques of convolutional coding, data interleaving, and error correction are addressed in detail.

In this paper, a class of periodic unipolar binary sequences is investigated for radio channel sharing among multiple users. The reported work offers an approach of distributed wireless 
access, particularly with interesting properties of individual performance guarantees. The system model is described in Section II. Sequence designs are investigated in Section III. For the enhancement of the system's allowable rate sum, new constructions are presented in Section IV. Section V gives the numerical studies of throughput performance. Finally, some concluding remarks are provided in Section VI.

\section{SYSTEM MODEL}

Following Massey's model of collision channel without feedback [7], a communication channel shared by a number of $M$ active users is divided into time slots of equal duration. Each active user follows a binary protocol sequence $W=$ $\{W(0), W(1), W(2), \ldots\}$ and transmits a packet at time slot $i$ if and only if $W(i)=1$. A user with the permission to transmit in a given time slot will transmit a data packet. Otherwise, it keeps silent. In this paper, we limit the discussion and assume that users know and align to slot boundaries. However, users are not required to synchronize to each other. For example, different users may have different transmission starting times. They are allowed to have a relative time offset between each other. Some practices of slot-synchronized operation and the corresponding details can be found in [23]-[26]. It is worth pointing out that, for full flexibility, one would consider eliminating the requirement of synchronized slot boundaries. It is, in fact, possible to do so and allow users to be completely unsynchronized [9], [22], [27]. However, for simplicity, in this paper, we will only focus on the slot-synchronized model.

At any time slot, a packet collision occurs if more than one user transmits at the same time. All transmitted packets in this duration are considered lost. Otherwise, the receiver can correctly receive its packet and decode the content. Discussions on sender and packet identification issues can be found in [9] and [17] for practical considerations. However, one can also consider the simple approach of requiring each packet to include a header, which contains the user identity and data index commonly defined in conventional medium-access control protocols [6]. If the payload of a packet is large enough, the cost of this overhead could be quite small.

To measure throughput performance, the effective rate of a user is defined as the fraction of packets that it can send without suffering any collision. In a random-access system, one is usually most interested in the symmetric case, in which all the users are signaling at the same rate [7]. Here, we will focus on the design for symmetric users. In this scenario, it is well known that the system capacity or effective rate sum of a slot-synchronized system will approach $1 / e$ as the number of active users $M$ tends to infinity. However, in addition to system throughput, it is also important to look at the service reliability for each individual user [28]. Related works in this tradition usually focus on the system throughput. However, it will be favorable to have a design that can support both system and individual performance stability such that one can always ensure the reliability of a communication channel for any of the active users, as long as the sum of allowable rates does not exceed a specified level.

\section{Multiple-Access Protocols}

An investigation of the class of linear congruence sequence [10] and its deployment for distributed multiple access is conducted here. To begin with, some definitions are required.

\section{A. Linear Congruence Sequence}

Let $W=\{W(i), i=0,1,2, \ldots\}$ be a binary sequence. Sequence $W$ can also be represented by indexing the positions at which it has a value of 1 , i.e., by $\left\{I_{W}(i), i=1,2, \ldots\right\}$, where $I_{W}(i)$ denotes the position at which the $i$ th entry of $1 \mathrm{~s}$ in $W$ appears. For a periodic binary sequence with period $L$, its duty factor [7] or proportional rate $r$ is defined as

$$
r=\frac{1}{L} \sum_{i=0}^{L-1} W(i) .
$$

Let $b$ and $l$ be two relatively prime integers with $0 \leq b<l$. The linear congruence sequence generated by $(b, l)$ can be expressed [17] as

$$
I_{W}(i)=i l+i b-\left\lfloor\frac{i b}{l}\right\rfloor l
$$

where $b$ is known as the key generator, and $i$ starts from 1 . The sequence generated by $(b, l)$ has a duty factor of $1 / l$. For $b>0$, it has a period of $l^{2}$. Subsequence $W=\{W(i), i=$ $\left.0,1, \ldots, l^{2}-1\right\}$ is known as the core pattern. Given $b=0$, by substitution, (2) may be reduced to

$$
I_{W}(i)=i l .
$$

Its minimum period is equal to $l$.

For example, let $b=1$ and $l=3$; following (2), $I_{W}$ is equal to $\{4,8,9, \ldots\}$, whereas the core pattern of $W$ is equivalent to $\{0,0,0,1,0,0,0,1,1\}$. It has a duty factor $r$ of one third and a period of nine.

Note that, for prime $l$, the set of linear congruence sequences is also known as prime sequences [11], which can be obtained from a Galois field $\mathrm{GF}(l)$ and represented [2] as

$$
\{0, b, 2 b, \ldots,(l-1) b\} \text { modulo } l
$$

where $0 \leq b \leq l-1$.

\section{B. Correlation Properties}

Let $W_{1}$ and $W_{2}$ be two binary sequences with common period $L$ and duty factors $r_{1}$ and $r_{2}$, respectively. For any relative time shift $s$, the Hamming cross-correlation function between $W_{1}$ and $W_{2}$ is defined as

$$
H_{W_{1}, W_{2}}(s)=\sum_{i=0}^{L-1} W_{1}(i) W_{2}(i+s)
$$

whereas the normalized function is denoted by

$$
\bar{H}_{W_{1}, W_{2}}(s)=\frac{1}{L} \sum_{i=0}^{L-1} W_{1}(i) W_{2}(i+s) .
$$




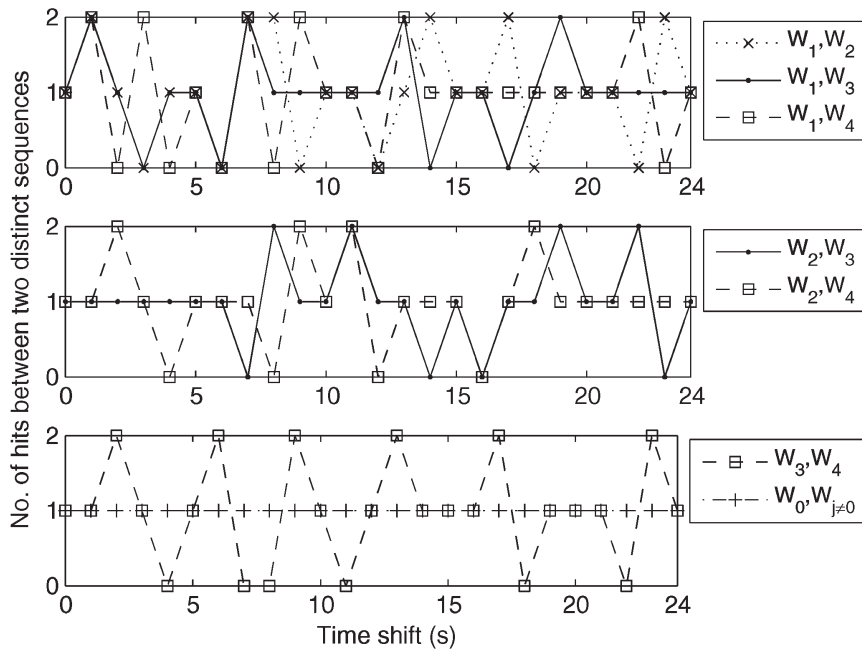

Fig. 1. Number of hits between any two distinct linear congruence sequences with relative time shift $s$ from 0 to 24 when $l=5$.

Let $l, b_{1}$, and $b_{2}$ be integers satisfying $0 \leq b_{1}<l, 0 \leq b_{2}<l$, $b_{1} \neq b_{2}$, respectively, and $\operatorname{HCF}\left(\left|b_{2}-b_{1}\right|, l\right)=1$; let $W_{1}$ and $W_{2}$ be the linear congruence sequences generated by $\left(b_{1}, l\right)$ and $\left(b_{2}, l\right)$, respectively. It is known [17] that

$$
\bar{H}_{W_{1}, W_{2}}(s) \leq 2 / l^{2}=2 r^{2}
$$

for any $0 \leq s<l^{2}$, where $r_{1}=r_{2}=r=1 / l$. For $b_{1}, b_{2}>0$, the equality of (7) holds at least once, and the upper bound is tight. In the special case when $b_{1}=0$ or $b_{2}=0$

$$
\bar{H}_{W_{1}, W_{2}}(s)=1 / l^{2}=r^{2} .
$$

For demonstration, let $l=5$ and $W_{i}$ be the sequence generated by $(i, l)$, where $0 \leq i \leq 4$. Here, $\operatorname{HCF}\left(\left|b_{i}-b_{j}\right|, 5\right)=1$, for any $i \neq j$. Following (2)

$$
\left[\begin{array}{l}
W_{1} \\
W_{2} \\
W_{3} \\
W_{4} \\
W_{0}
\end{array}\right]=\left[\begin{array}{l}
0000010000010000010000011 \\
0000001000000101000000101 \\
0000000100100000001001001 \\
0000000010001000100010001 \\
0000100001000010000100001
\end{array}\right] .
$$

The number of coincidences or hits between any two distinct sequences or rows in (9) is depicted in Fig. 1 for all the relative time shifts $0 \leq s<l^{2}$. The result illustrates that

$$
0 \leq H_{W_{i}, W_{j \neq i}}(s) \leq 2
$$

In particular, $H_{W_{0}, W_{j}}(s)=1$ for any $0<j<l$.

\section{Family of Protocol Sequences}

By the aforementioned results, a family of periodic protocol sequences can be formulated here. To satisfy the condition for the performance of (7) and (8) and have full flexibility in choosing key generator $b$, we simply employ prime $l$. In the following, $p$ is used to denote prime $l$. Consequently, the considered sequences may also refer to prime sequences.
Definition 1: For any prime $p$, a family of periodic binary sequences $\mathbf{F}_{p}$ is defined as the set of linear congruence sequences generated by $(b, p)$, where $0 \leq b<p$.

Note that the sequences in $\mathbf{F}_{p}$ have a minimum common period $L=p^{2}$, and each has a duty factor $r_{i}=1 / p$. Following Definition 1, a nontotally blockage property of a set of the aforementioned sequences can accordingly be established. The details are given here.

Consider a collision channel without feedback, in which multiple active users need to be supported in a distributed manner, and over a long time horizon, occasional data loss is not a serious problem, or it can be recovered by some channel coding [8], [22]; set $\mathbf{F}_{p}$ could be used to define protocol sequences for data transmissions. For any finite subset of $\mathbf{F}_{p}$ denoted by $\mathbf{W}=\left\{W_{i}, i=0, \ldots, N-1\right\}$, where $N$ denotes the cardinality of $\mathbf{W}$, provided that

$$
\sum_{j=0, j \neq i}^{N-1} r_{j} \equiv\left(\sum_{j=0}^{N-1} r_{j}\right)-r_{i} \leq \frac{1}{2}
$$

which refers to the sum of the duty factors of the active users, excluding $r_{i}$, by (10) and (11), one can find that the total number of collisions to $W_{i}$ can be expressed as

$$
\sum_{j=0, j \neq i}^{N-1} \sum_{t=0}^{L-1} W_{i}(t) W_{j}\left(t+s_{j}\right) \leq \begin{cases}\left\lfloor\frac{1 / 2}{1 / p}\right\rfloor \times 2, & p>2 \\ \frac{1 / 2}{1 / p} \times 1, & p=2\end{cases}
$$

which is always strictly less than $p$ or, equivalently, $r_{i} L$, for prime $p$ and any combination of relative time shifts $s_{j}$ of $W_{j}$, with reference to $W_{i}$. This implies that there cannot be enough collisions to completely block any particular user in any period $L$, i.e., $p^{2}$, as long as the sum of the duty factors of other active users $\left\{r_{j \neq i}\right\}$ does not exceed one half, even when the relative time shifts among users may change from time to time for different reasons, for example, user mobility or time-varying propagation delay.

Similarly, one can show [28] that, as long as $\sum_{j=0}^{N-1} r_{j} \leq$ $1 / 2$, for each user in the preceding set, at least two transmission packets within the common period encountered, i.e., $p^{2}$ slots, will not be blocked. This unsuppressibility [17] property is helpful in ensuring that, even in the worst case, all the users can successfully transmit information to a guaranteed amount in every time period in a distributed random-access manner, even without the requirements of user synchronization and packet retransmissions.

However, without loss of generality, the bound on the allowable rate sum stated in (11) is not tight enough. One can have examples of sequence sets in which the aforementioned nontotally blockage holds while (11) is violated. A natural followup is to enhance the maximum allowable rate sum and design a corresponding sequence set that possesses the desired user unsuppressibility. New constructions are given in the following. 


\section{New Constructions}

In this section, designs of periodic binary sequences with smaller normalized cross correlation are presented. They are helpful in enhancing the allowable rate sum.

\section{A. Enhancement of Allowable Rate Sum}

Recall (7) and (10); it is observed that

$$
H_{W_{i}, W_{j}}(s)=\{0,1,2\}
$$

where $\bar{H}_{W_{i}, W_{j}}(s) \leq 2 r^{2}$ for $i \neq j$. This could be a hint as to the construction of a new set of codewords with $\bar{H}_{W_{i}, W_{j}}(s)=r^{2}$ based on an inspection of the following property [29]:

$$
\lambda_{\text {avg }}\left(W_{i}, W_{j}\right) \triangleq \sum_{s=0}^{L-1} \bar{H}_{W_{i}, W_{j}}(s)=r_{i} r_{j} L
$$

which indicates that the average number of hits between any two sequences $W_{i}$ and $W_{j}$, with respective duty factors $r_{i}$ and $r_{j}$ over their common period $L$, is equal to $r_{i} r_{j} L$. This is due to the fact that each of the $r_{i} L$ 1s in $W_{i}$ will meet the $r_{j} L 1 \mathrm{~s}$ in $W_{j}$ when $s$ shifts from 0 to $1,2, \ldots$, up to $L-1$ stepwise. Following the definitions of (5) and (6), by (14), one can have the following fact:

$$
\sum_{s=0}^{L-1} H_{W_{i}, W_{j}}(s) \equiv L \cdot \sum_{s=0}^{L-1} \bar{H}_{W_{i}, W_{j}}(s)=r_{i} L \cdot r_{j} L .
$$

For example, when $p=5$, as indicated in Fig. 1, $\sum_{s=0}^{L-1} H_{W_{i}, W_{j}}(s)=25$, where $\lambda_{\text {avg }}\left(W_{i}, W_{j}\right)=1$. Here, $r_{i}=$ $1 / p=1 / 5$, and $L=p^{2}=25$.

The average number of hits between two sequences is, in fact, equal to 1 after averaging out over all the relative time shifts $0 \leq s \leq L-1$. By (14), it is clear that the frequency of " $H_{W_{i}, W_{j}}(s)=2$ " is the same as that of " $H_{W_{i}, W_{j}}(s)=$ 0 ." There is no bias. $\lambda_{\operatorname{avg}}\left(W_{i}, W_{j}\right)$ is not dominated by " $H_{W_{i}, W_{j}}(s)=2$." The scenario is sometimes "bad," i.e., equal to 2 , but sometimes "good," i.e., equal to 0 . Otherwise, it is "fair" and just in between, i.e., equal to 1 .

By the preceding observation, one way to obtain a set of periodic binary sequences with $\bar{H}_{W_{i}, W_{j}}(s)=r_{i} r_{j}$ for any relative shift $s$ can be conducted by averaging out all the scenarios. More explicitly, we can concatenate the original prime sequences with their shifted versions to take and combine all the effects due to the relative time shifts. As a result, one can get the average performance, i.e., on average, one collision with another sequence per $p$ packets transmitted over their encountered common period, and maintain it at this cross correlation for any $s$. Thus, as long as

$$
\sum_{j=0}^{N-1} r_{j} \leq 1
$$

there cannot be enough collisions to completely block any particular sequence, no matter how the protocol sequences are shifted with respect to one another. It can be shown by the following upper bound that the number of collisions to sequence $i$ due to all the other collisions is strictly less than the number of packets it transmits $L / p$ in common period $L$. Following (5) and (16)

$$
\begin{aligned}
\sum_{j=0, j \neq i}^{N-1} & \sum_{t=0}^{L-1} W_{i}(t) W_{j}\left(t+s_{j}\right) \equiv \sum_{j=0, j \neq i}^{N-1} H_{W_{i}, W_{j}}\left(s_{j}\right) \\
= & \sum_{j=0, j \neq i}^{N-1}\left(\frac{L}{p} \cdot \frac{1}{p}\right) \leq \frac{1-(1 / p)}{1 / p} \cdot \frac{L}{p^{2}}=\frac{L}{p}-\frac{L}{p^{2}}<\frac{L}{p}
\end{aligned}
$$

or, equivalently, $r_{i} L$, where $\sum_{j \neq i} r_{j} \leq 1-1 / p$.

Consequently, by (17), the effective rate of a user with a duty factor of $1 / p$ has a lower bound of $1 / p^{2}$. However, it is worth pointing out that the actual throughput could be much higher. The numerical studies in Section V show more of the details.

\section{B. Construction 1}

For the aforementioned properties of (16) and (17), a new design is necessary. The details are given here. Let us start with $p=3$ to describe the construction. Since, by $(8), \bar{H}_{W_{0}, W_{1}}(s)=$ $\bar{H}_{W_{0}, W_{2}}(s)=r^{2}=1 / 9$, we first take $W_{0}$ and $W_{2}$ as codewords in our sequence set. Otherwise, one can choose the pair $W_{0}$ and $W_{1}$ instead. The result is equivalent.

By (7), $\max _{s} \bar{H}_{W_{1}, W_{2}}(s)=2 / 9$. Thus, if $W_{0}, W_{1}$, and $W_{2}$ are simultaneously used, $W_{1}$ (or $W_{2}$ ) will be completely blocked by $W_{2}$ and $W_{0}$ ( $W_{1}$ and $W_{0}$, respectively) in the worst case. This can be observed in many examples and has been verified in experiments [30]. Fig. 2 in Section V can show the phenomenon in general. The details will be given later.

To keep all the codewords with $\bar{H}_{W_{i}, W_{j \neq i}}(s)=1 / 9$, one can use (15) as the basis and follow the idea of "averaging out" to have the following sequence set:

$$
\left[\begin{array}{l}
W_{1}^{(1)} \\
W_{2}^{(1)} \\
W_{0}^{(1)}
\end{array}\right]=\left[\begin{array}{llll}
W_{1, \theta=0} & W_{1, \theta=1} & \cdots & W_{1, \theta=8} \\
W_{2, \theta=0} & W_{2, \theta=0} & \cdots & W_{2, \theta=0} \\
W_{0, \theta=0} & W_{0, \theta=0} & \cdots & W_{0, \theta=0}
\end{array}\right]
$$

where $W_{i, \theta=n}$ refers to a version of $W_{i}$ shifted by $n$ bits toward the left, and the superscript on $W_{i}^{(1)}$ is used to label the new codeword by Construction 1. For example, since $W_{1, \theta=0} \equiv$ $W_{1}=\{000100011\}, W_{1, \theta=1}=\{001000110\}$. Note that the new codewords need to be much longer.

Based on (18), one can find that, for any relative shift $s$

$$
\begin{aligned}
\bar{H}_{W_{1}^{(1)}, W_{2}^{(1)}}(s) & \equiv \frac{1}{p^{4}} \sum_{i=0}^{p^{4}-1} W_{1}^{(1)}(i) W_{2}^{(1)}(i+s) \\
& =\frac{1}{p^{4}} \sum_{n=0}^{p^{2}-1} \sum_{i=0}^{p^{2}-1} W_{1, \theta=n}(i) W_{2, \theta=0}(i+s) \\
& =\frac{1}{p^{4}} \sum_{n=0}^{p^{2}-1} \sum_{i=0}^{p^{2}-1} W_{1}(i) W_{2}(i+s-n) \\
& =\frac{1}{p^{4}} \sum_{n=0}^{p^{2}-1}\left(p^{2} \cdot \bar{H}_{W_{1}, W_{2}}(s-n)\right) \\
& =\frac{1}{p^{4}} \times p^{2} \times 1=\frac{1}{p^{2}}
\end{aligned}
$$


since, by (14), $\sum_{n=0}^{p^{2}-1} \bar{H}_{W_{1}, W_{2}}(s-n)=1$, where $r_{i}=1 / p$, and $L=p^{2}$. The result is independent of $s$.

By (8), similarly, one can also find that

$$
\bar{H}_{W_{0}^{(1)}, W_{1}^{(1)}}(s)=\bar{H}_{W_{0}^{(1)}, W_{2}^{(1)}}(s)=1 / p^{2} .
$$

Therefore, we can claim that $\left\{W_{0}^{(1)}, W_{1}^{(1)}, W_{2}^{(1)}\right\}$ is a set of sequences with normalized Hamming cross correlation equal to $r^{2}$ for any relative time shift $s$. Even when all the codewords are simultaneously used and, thus, the rate sum is equal to 1 , the nontotally blockage property holds.

For $p=5$, to keep all the codewords with $\bar{H}_{W_{i}, W_{j \neq i}}(s)=$ $1 / 25$, a new set of protocol sequences can similarly be constructed as the following:

$$
\left[\begin{array}{l}
W_{1}^{(1)} \\
W_{2}^{(1)} \\
W_{3}^{(1)} \\
W_{4}^{(1)} \\
W_{0}^{(1)}
\end{array}\right]=\left[\begin{array}{ccc}
{\left[W_{1, \theta=0}\right.} & \cdots & \left.W_{1, \theta=24}\right]_{25 \times 25} \\
{\left[\left[W_{2, \theta=0}\right]_{25}\right.} & \cdots & \left.\left[W_{2, \theta=24}\right]_{25}\right]_{25} \\
{\left[W_{3, \theta=0}\right]_{25 \times 25}} & \cdots & {\left[W_{3, \theta=24}\right]_{25 \times 25}} \\
{\left[W_{4, \theta=0}\right]_{25 \times 25 \times 25}} \\
{\left[W_{0, \theta=0}\right]_{25 \times 25 \times 25}}
\end{array}\right]
$$

where $\left[W_{i}\right]_{k}$ refers to a concatenation of $k$ copies of $W_{i}$ consecutively. The incentive is to extend the original sequences such that, for any two distinct sequences, all the shift combinations are included. Consequently, no matter how the shift is, the resultant cross correlation always contains all the good, bad, and fair scenarios. Thus, by (14)

$$
\bar{H}_{W_{i}^{(1)}, W_{j}^{(1)}}(s)=1 / p^{2} .
$$

The proof is very similar to that in (19) and thus is omitted. One can conclude that, even when all the codewords in (21) are used simultaneously with active users of rate sum equal to 1 , the nontotally blockage property still holds.

Following the definition of prime sequence, for each $p$, a set of corresponding sequences with $\bar{H}_{W_{i}, W_{j \neq i}}(s)=1 / p^{2}$ can be constructed in an iterative way. It is worth pointing out that the proposed idea of "averaging out" is generally applicable to any sequence set as long as the property of (14) holds. Similar constructions and results are achievable.

On the other hand, as previously mentioned, the new sequence needs to be much longer than the original one. Let $L_{p}^{(1)}$ be the length of codewords in Construction 1 for the set of $p$. Since $W_{0}^{(1)}$ requires only a simple concatenation, we have

$$
L_{p}^{(1)}=\left(p^{2}\right)^{p-1}=p^{2(p-1)}
$$

For example, let $p=3$ and $L_{3}^{(1)}=3^{4}=81$. Accordingly, $L_{5}^{(1)}=25^{4}$. Note that $L_{p}^{(1)}$ could be very large as (23) is exponentially increasing. For example, when $p=7, L_{7}^{(1)}=7^{12}$. In a channel of $250 \mathrm{~kb} / \mathrm{s}$, it will take about $5.54 \times 10^{4} \mathrm{~s}$ to complete one sequence cycle. This could be a problem for applications. A natural follow-up is to look for a shorter required codeword length. Construction 2 is thus proposed here.

\section{Construction 2}

In the previous construction, by the observation of (14), we may have collected too many copies of the variants or shifted versions of the original sequences to achieve the result of averaging out all the cross correlations. However, some concatenations are unnecessary.

By the cross-correlation properties of linear congruence sequences [17], the following expression can be established:

$$
\sum_{s=0}^{p-1} \bar{H}_{W_{i}, W_{j \neq i}}(s \cdot p+t)=1 / p
$$

for any integer $0 \leq t<p$. For example, given $p=3$

$$
\begin{aligned}
\sum_{\tau=0,3,6} \bar{H}_{W_{i}, W_{j}}(\tau) & =\sum_{\tau=1,4,7} \bar{H}_{W_{i}, W_{j}}(\tau) \\
& =\sum_{\tau=2,5,8} \bar{H}_{W_{i}, W_{j}}(\tau)=\frac{1}{3}
\end{aligned}
$$

This implies that a design that contains concatenations with relative time shift combinations over the set $\{0, p$, $2 p, \ldots, p(p-1)\}$ is, in fact, sufficient in characterizing the overall cross-correlation performance and representing all the good, bad, and fair scenarios.

For $p=3$, the new construction is given by

$$
\left[\begin{array}{l}
W_{1}^{(2)} \\
W_{2}^{(2)} \\
W_{0}^{(2)}
\end{array}\right]=\left[\begin{array}{lll}
W_{1, \theta=0} & W_{1, \theta=3} & W_{1, \theta=6} \\
W_{2, \theta=0} & W_{2, \theta=0} & W_{2, \theta=0} \\
W_{0, \theta=0} & W_{0, \theta=0} & W_{0, \theta=0}
\end{array}\right] .
$$

Following (26), by (24), the normalized cross correlation can be expressed as

$$
\begin{aligned}
\bar{H}_{W_{1}^{(2)}, W_{2}^{(2)}}(s) & =\frac{1}{p^{3}} \sum_{n=0}^{p-1} \sum_{i=0}^{p^{2}-1} W_{1, \theta=n p}(i) W_{2, \theta=0}(i+s) \\
& =\frac{1}{p^{3}} \sum_{n=0}^{p-1} \sum_{i=0}^{p^{2}-1} W_{1}(i) W_{2}(i+s-n p) \\
& =\frac{1}{p^{3}} \sum_{n=0}^{p-1}\left(p^{2} \cdot \bar{H}_{W_{1}, W_{2}}(s-n p)\right) \\
& =\frac{1}{p^{3}} \times p^{2} \times \frac{1}{p}=\frac{1}{p^{2}} .
\end{aligned}
$$

Similarly, by (8), it can be shown that $\bar{H}_{W_{0}^{(2)}, W_{1}^{(2)}}(s)=$ $\bar{H}_{W_{0}^{(2)}, W_{2}^{(2)}}(s)=1 / p^{2}$. Hence, even when all the codewords $W_{0}^{(2)}, W_{1}^{(2)}$, and $W_{2}^{(2)}$ are employed at the same time and, thus, the rate sum of active users is equal to 1 , the nontotally blockage property is still maintained. 
For $p=5$, the construction can iteratively be formulated as $\left[\begin{array}{l}W_{1}^{(2)} \\ W_{2}^{(2)} \\ W_{3}^{(2)} \\ W_{4}^{(2)} \\ W_{0}^{(2)}\end{array}\right]=\left[\begin{array}{cccc}{\left[W_{1, \theta=0}\right.} & W_{1, \theta=5} & \cdots & \left.W_{1, \theta=20}\right]_{5 \times 5} \\ {\left[\left[W_{2, \theta=0}\right]_{5}\right.} & {\left[W_{2, \theta=5}\right]_{5}} & \cdots & \left.\left[W_{2, \theta=20}\right]_{5}\right]_{5} \\ {\left[W_{3, \theta=0}\right]_{5 \times 5}} & \cdots & {\left[W_{3, \theta=20}\right]_{5 \times 5}} \\ {\left[W_{4, \theta=0}\right]_{5 \times 5 \times 5}} \\ {\left[W_{0, \theta=0}\right]_{5 \times 5 \times 5}}\end{array}\right]$.

Consequently, $\bar{H}_{W_{i}^{(2)}, W_{j}^{(2)}}(s)=1 / p^{2}$ for any $j \neq i$. Even when $\sum_{j} r_{j}=1$, the nontotally blockage property holds.

Codewords of different $p$ 's can be constructed in an iterative way and are given by (29), shown at the bottom of the page.

Denote the length of codewords in Construction 2 by $L_{p}^{(2)}$. Following (29)

$$
L_{p}^{(2)}=p^{2} \times p^{p-2}=p^{p} .
$$

For example, $L_{3}^{(2)}=27$, whereas $L_{5}^{(2)}=3125$. Comparing (30) with (23), their ratio is given by

$$
\frac{L_{p}^{(2)}}{L_{p}^{(1)}}=\frac{p^{p}}{p^{2(p-1)}}=\frac{1}{p^{p-2}}
$$

which indicates a big reduction in the sequence length by Construction 2 when compared with Construction 1 . To give a demonstration, when $p=7, L_{7}^{(2)}=7^{7}$. In a channel of $250 \mathrm{~kb} / \mathrm{s}$, it takes about $3.29 \mathrm{~s}$ to complete one cycle. This is much smaller than $5.54 \times 10^{4} \mathrm{~s}$ in Construction 1 and could be acceptable for certain applications.

However, it should be noted that $L_{p}^{(2)}$ is still exponentially increasing, despite it being much smaller than $L_{p}^{(1)}$. Consequently, the number of active users allowed will be limited if long codewords are not suitable. A further improvement on the design of codewords will be favorable and left open. Generally, there are tradeoffs between different performance criteria.

\section{Throughrut Evaluation}

The effective rates of users following the protocol sequences by Constructions 1 and 2 in the slot-synchronized collision channel are investigated. Both individual and system throughputs are measured. In the simulation study, we assume that there are $M$ active users, and each will transmit at a rate of $1 / p$ of the channel bandwidth. The protocol sequences are used to specify their channel access permission time. Accordingly, a user will transmit a packet at the time slot when its proto- col sequence refers to "1." Otherwise, it keeps silent. Since users are unsynchronized to each other and may have different transmission starting times, there will be various combinations of relative time shifts. In the simulation, we assume that the relative time shift $s$ between two users is uniformly distributed in their encountered minimum period.

User throughputs are measured when the system is fully saturated, i.e., $\sum_{i=1}^{M} r_{i}=1$, where $M=p$, and $r_{i}=1 / p$. Data are evaluated after a simulation of $10^{5}$ runs for various time shift combinations. Results obtained show that the protocol sequences from Constructions 1 and 2 have the same performance, as expected. Thus, in the following, only one set of the data is plotted. However, the plotted results can represent both of them and are simply labeled as "New."

Fig. 2 shows the individual throughputs of users with respect to different $p$ 's. Each throughput is measured with respect to its encountered minimum period. The minimum, mean, and maximum values of individual throughputs obtained from the new constructions are plotted and compared with those obtained from prime sequences and a random-access scheme. In the random access, it is assumed that, at each time slot, a user will transmit a packet with probability equal to its duty factor $1 / p$ and in uniform distribution. The effective rate of a user in the random-access scheme is measured in periods that are the same as those in prime sequences with respect to different $p$ 's.

Comparing the performance of the new construction, prime sequences, and random access, the means of their individual throughputs are the same. This is expected as long as the mean is taken by averaging the throughputs of users with uniformly distributed relative time shifts. One can also observe from Fig. 4 that they have the same average system throughput as well. The range of individual throughputs from simulation is indicated by [Min, Max] and shown in Fig. 2, whereas the standard deviation (SD) is plotted in Fig. 3 to indicate the performance fluctuations. As shown in Fig. 2, a user with prime sequence can completely be blocked by other active users in the collision channel, and the minimum individual throughput is equal to zero. The random access has the same problem. Meanwhile, the new construction has demonstrated its robustness that there cannot be enough collisions to completely block any particular sequence, no matter how they are shifted with respect to one another, which can eliminate the risk of being completely blocked. More importantly, both Figs. 2 and 3 have shown that the new construction has a zero variance of individual throughputs among all the users for any relative time shift combination in the simulations. In other words, users

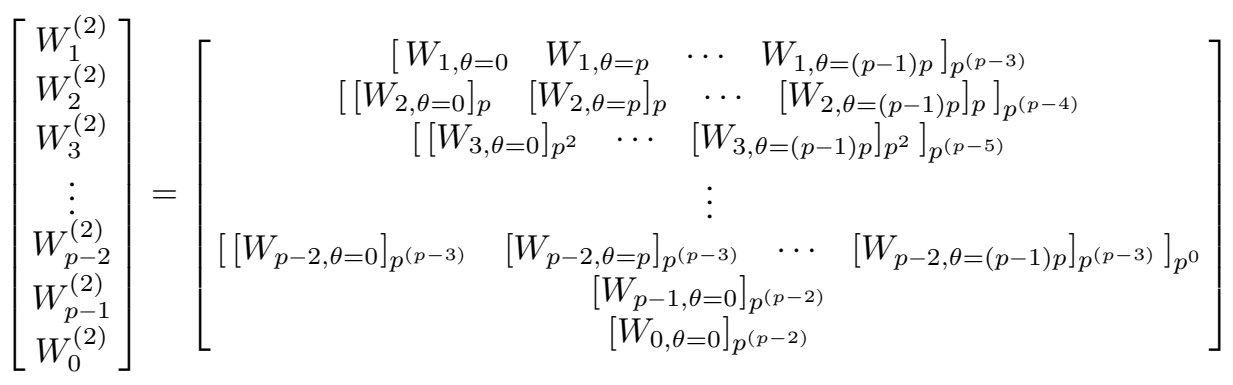




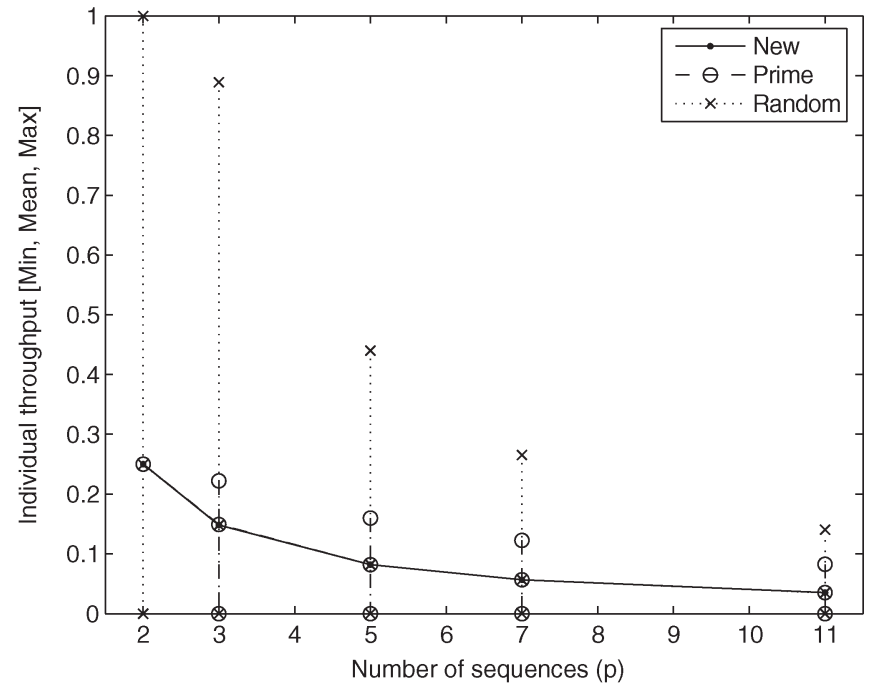

Fig. 2. Minimum, mean, and maximum individual throughputs from the simulation are shown. The duty factor of a user is equal to $1 / p$.

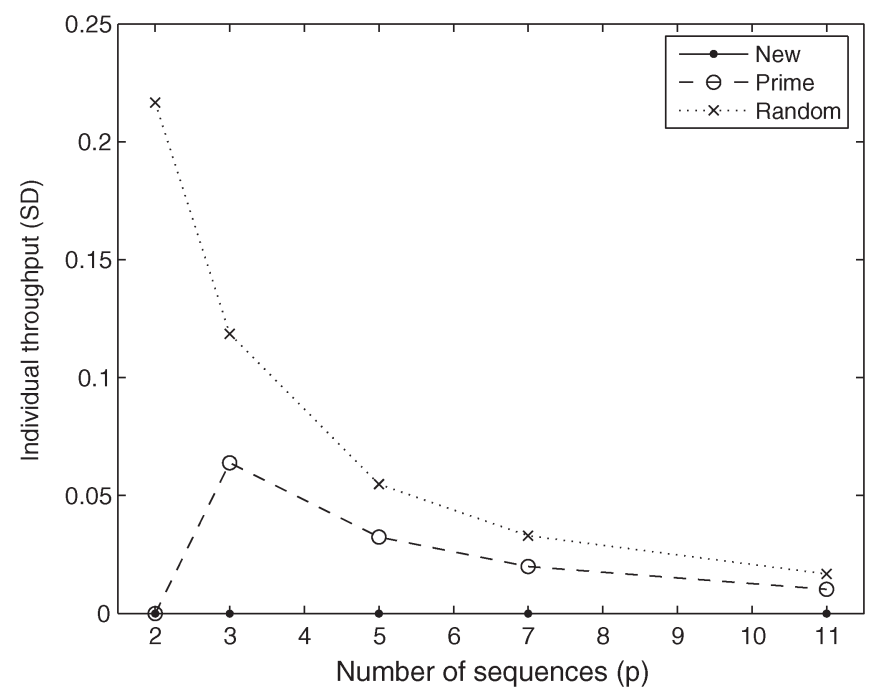

Fig. 3. SDs of individual throughputs are shown for reference. The result is associated with Fig. 2.

have the same and shift-invariant individual throughput over their periods. This indicates an extremely stable throughput performance, in contrast to the highly fluctuated performance in the prime sequence and random-access schemes. The result is helpful in providing a deterministic performance guarantee and could be particularly useful and convenient for channel coding purposes.

It is worth noting that the new construction will have a longer sequence period $p^{p}$ than that of prime sequence $p^{2}$ in general. In addition, in the prime sequence and random-access schemes, the probability of obtaining zero individual throughput could be smaller when the throughput is measured over a longer period. With reference to the study in [30], the random-access scheme could obtain a smaller variance of individual throughput, whereas the difference to prime sequences is insignificant. However, note that a user in these two schemes always has a nonzero probability of being completely blocked. In addition, as shown in Fig. 2, although the average individual throughputs

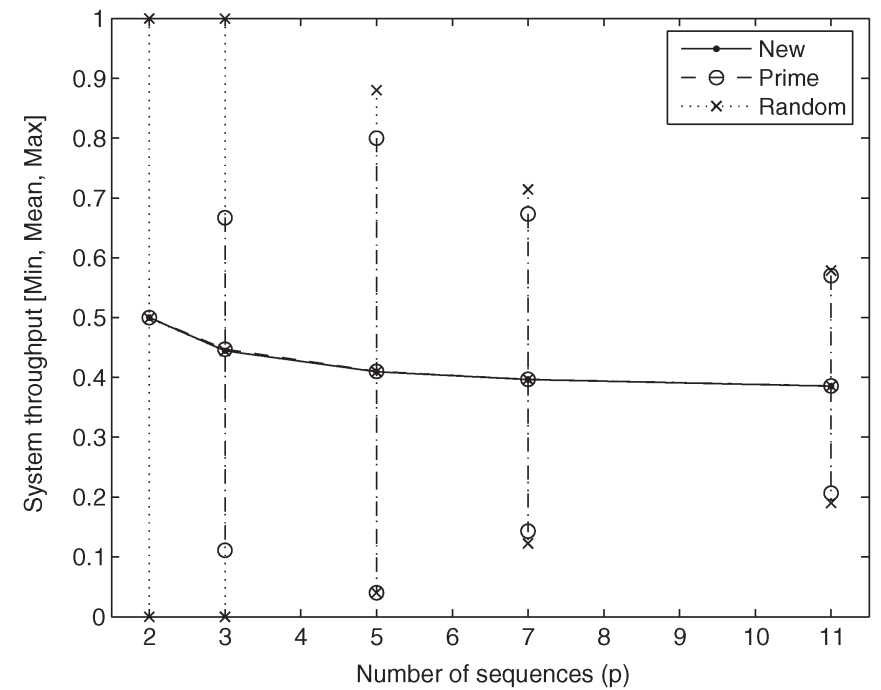

Fig. 4. Minimum, mean, and maximum system throughputs from the simulation are shown with respect to different prime $p$ 's.

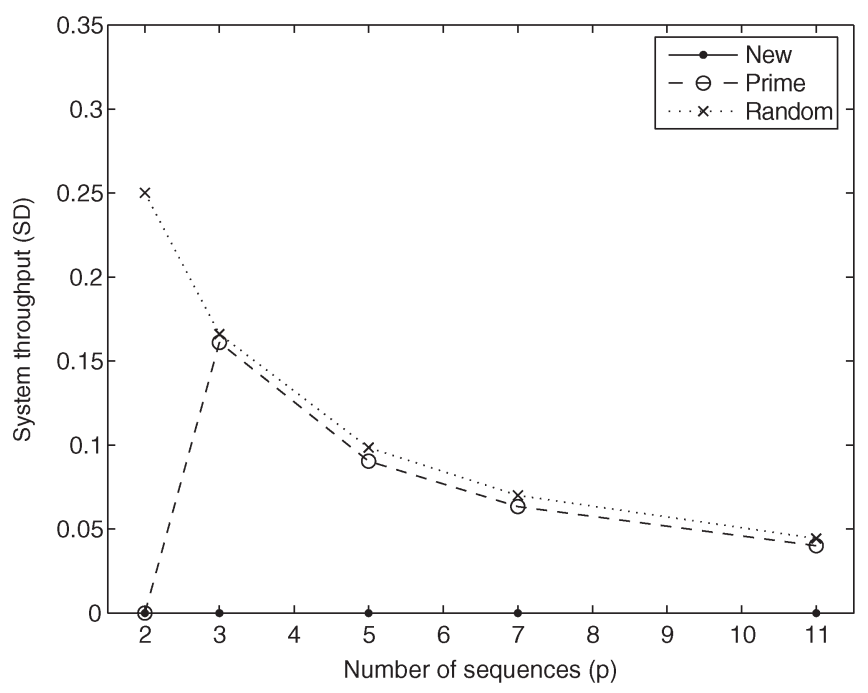

Fig. 5. SDs of system throughputs are shown for reference. The result is associated with Fig. 4.

in these three schemes are the same, the new construction has guaranteed a generally higher minimum throughput.

Figs. 4 and 5 show the system throughputs and their SDs, respectively. As expected, the system average throughput tends to $e^{-1}$ as $p$ goes to infinity [24]. In addition to the average performance, it is observed that the new construction has a zero variance of system throughput as well. However, as shown in Figs. 4 and 5, both the prime sequence and random-access schemes have high fluctuations. The result shows that the new construction supports a very robust and stable system performance. Meanwhile, it is capable of ensuring the nontotally blockage of each individual user.

Moreover, we conduct a performance comparison between the protocol sequence from Construction 2 and that from Massey and Mathys [9]. They have the same sequence period in this model. Fig. 6 indicates the similarity and difference. Results show that they have the same average individual and system throughputs when the effective rates are measured over 


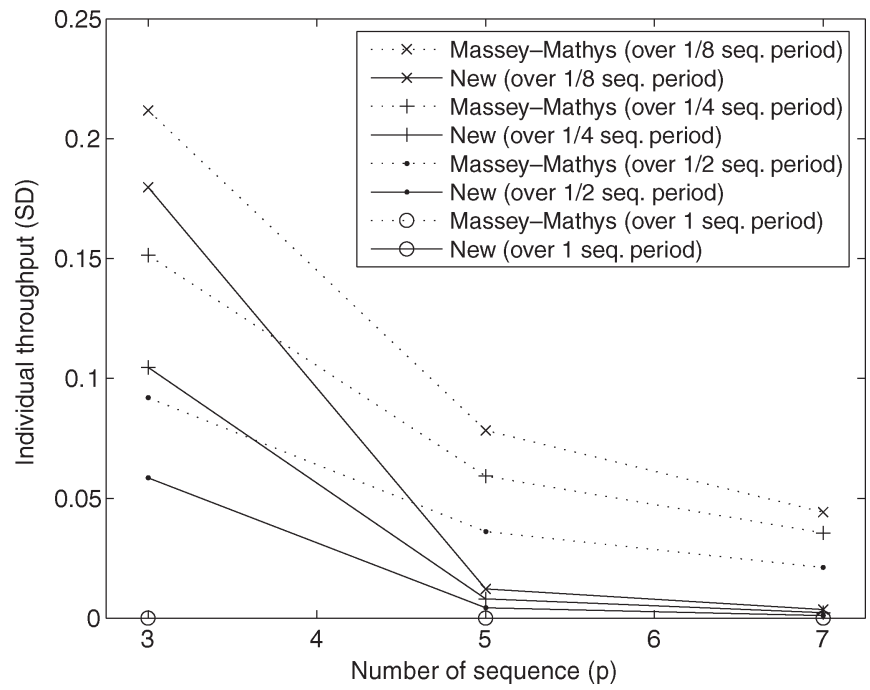

Fig. 6. SDs of individual throughputs measured over one eighth, one fourth, one half, and one sequence periods are plotted.

complete cycles of their minimum periods. In addition, it is observed that the construction in [9] can also achieve zero variance in individual and system throughputs as well. However, when effective throughputs are considered in partial periods for practical considerations as the period is exponential, there is a significant difference between the two schemes. Fig. 6 shows their SDs of individual throughputs measured over one eighth, one fourth, one half, and one sequence periods. We can see that the new construction outperforms and has a much smaller performance fluctuation in partial periods. However, it should be noted that [9] is more focused on determining the general capacity regions and finding out interesting techniques of packet recovery and decimation decoding skills for sender identification.

Note that individual and system throughputs of the new constructions can be expressed in closed form. Appendix A reports the analytical result for reference. One can find that they meet the capacity bound given in [9] under the slotsynchronized access model as well. In addition, it can be found that, for the symmetric rate case, the minimum necessary length of the protocol sequences with zero-variance individual and system throughputs is, in fact, equal to $p^{p}$. A proof is provided in Appendix B. Generally speaking, there is always a tradeoff between the period length of protocol sequences and the variance of throughput performance. If a higher variance of throughput performance is allowed, the length of the protocol sequences could be reduced.

\section{CONCLUding REMARK}

This paper has investigated a class of linear congruence sequences with interesting cross-correlation properties in defining new multiple-access protocols. Their reported characteristics have resulted in new constructions, which can enhance the system's allowable rate sum while maintaining user unsuppressibility. Thus, no matter how the protocol sequences are shifted with respect to one another, no one will be completely blocked.
Furthermore, it is found that the new design has zero variance in their periodic individual and system throughputs, which corresponds to a very stable service and deterministic guarantees. When comparing the partial period throughput performance with [9], the new construction is better and has a much smaller fluctuation. Results have reflected its performance stability, robustness, and, particularly, deterministic guarantees.

Moreover, the protocol sequences have some inherent properties that are suitable for applications in certain wireless sensor and ad hoc networks. They do not require complicated processing, such as a backoff algorithm, random number generation, and strict user synchronization. Since the protocol sequences are deterministic and periodic, a receiver does not require continuous monitoring of the channel and can explicitly locate arrival packets and the sender's transmission time by knowledge of the protocol sequence. This can be more energy efficient. In addition, by the periodicity of the sequence, it is, in fact, possible to avoid some transmission collisions in a cooperative manner for throughput enhancement when concerned [30].

On the other hand, note that, in the current design, the sequence period needs to be exponentially increasing. A tradeoff between the required length and throughput performance is possible. There could be some new solutions. In addition, the result presented here may also lead to some interesting and potential applications in different communication systems.

\section{APPENDIX A \\ EfFective Rates of The Sequence SeT}

In a system of symmetric rate users with protocol sequences from Construction 1 and each with duty factor $r_{j}=1 / p$, the individual throughput $\tilde{r}_{j}$ can be expressed as

$$
\tilde{r}_{j}=\sum_{i=1}^{p}(1 / p)^{i}(-1)^{i-1}\left(\begin{array}{c}
p-1 \\
i-1
\end{array}\right)
$$

for all $j$, whereas the system throughput is equal to

$$
\begin{aligned}
\sum_{j=0}^{p-1} \tilde{r}_{j} & =p \sum_{i=1}^{p}(1 / p)^{i}(-1)^{i-1}\left(\begin{array}{c}
p-1 \\
i-1
\end{array}\right) \\
& =\sum_{i=1}^{p}(1 / p)^{i-1}(-1)^{i-1}\left(\begin{array}{c}
p-1 \\
i-1
\end{array}\right) \\
& =\sum_{i=0}^{p-1}(1 / p)^{i}(-1)^{i}\left(\begin{array}{c}
p-1 \\
i
\end{array}\right) \\
& =\sum_{i=0}^{p-1}(-1 / p)^{i}(1)^{(p-1)-i}\left(\begin{array}{c}
p-1 \\
i
\end{array}\right) \\
& =(1-1 / p)^{p-1} .
\end{aligned}
$$

It is found that the result of (33) is consistent with the symmetric capacity bound of the $M$-user collision channel without feedback [9]

$$
C_{\mathrm{sym}}=\left(1-\frac{1}{M}\right)^{M-1}
$$


with corresponding achievable rate point

$$
\left(C_{\mathrm{sym}} / M, C_{\mathrm{sym}} / M, \ldots, C_{\mathrm{sym}} / M\right)
$$

which can be expressed as (32), for each individual user.

The proof of (32) for Construction 1 can be done similarly to that of in [9, Lemma 2]. The details are given here. Consider the generated codewords in a binary matrix whose rows correspond to the protocol sequences, due to the sequence concatenation of all the combinations in Construction 1; no matter how the protocol sequences are cyclically shifted, it always results in a permutation of the columns. Thus, the resultant matrix will contain the same number of collisions and successful transmissions, regardless of the time offsets. Due to the symmetry, similarly to (14), the number of commonly overlapped 1 s among a number of $k$ protocol sequences, for any $2 \leq k \leq p$, is equal to

$$
\begin{array}{r}
\frac{1}{L^{k}} \sum_{s_{k}=0}^{L-1} \ldots \sum_{s_{2}=0}^{L-1} \sum_{i=0}^{L-1}\left[W_{1}(i) W_{2}\left(i+s_{2}\right)\right. \\
\left.\cdots W_{k}\left(i+s_{k}\right)\right] \\
=\frac{(L / p)^{k}}{L^{k}}=\frac{1}{p^{k}}
\end{array}
$$

where $s_{j}$ is used to denote the relative time shift of $W_{j}$, with reference to $W_{1}$. In addition, the result of (36) is independent of $j$ and, thus, is applicable to all the users. This leads to the expression of (32). Consequently, there is a zero-variance individual throughput among all of them. At the same time, the variance of system throughput is equal to zero as well.

A mathematical proof of (32) for Construction 2 is not available here due to the complexity, even if, conceptually, Construction 2 is just inherited from Construction 1 with (24). However, all the numerical results obtained from Construction 2 agree with (32) and (33).

\section{APPENDIX B Minimum NeCESSARY LENGTH}

Consider a set of periodic unipolar binary sequences $\left\{S_{1}, S_{2}, \ldots, S_{p}\right\}$ of symmetric rate $1 / p$ with common period $L$. Each of them contains $L / p 1$ s.

If the number of coincidences between two sequences, for example, $S_{1}$ and $S_{2}$, is shift invariant for any $0 \leq s \leq L-1$

$$
\sum_{s=0}^{L-1} H_{S_{1} S_{2}}(s)=L \times H_{S_{1} S_{2}}(s) .
$$

Similarly to (14), since each of the $L / p 1 \mathrm{~s}$ in $S_{1}$ will meet the $L / p 1 \mathrm{~s}$ in $S_{2}$ when $s$ shifts from 0 to $1,2, \ldots$, up to $L-1$

$$
\sum_{s=0}^{L-1} H_{S_{1} S_{2}(s)} \equiv \sum_{s=0}^{L-1} \sum_{i=0}^{L-1} S_{1}(i) S_{2}(i+s)=\frac{L}{p} \times \frac{L}{p}
$$

By (37) and (38)

$$
H_{S_{1} S_{2}}(s)=\frac{(L / p) \times(L / p)}{L}=\frac{L}{p^{2}}
$$

i.e., two sequences will meet at $L / p^{2}$ times.
Let us consider the number of common coincidences of three sequences, for example, $S_{1}, S_{2}$, and $S_{3}$, which can be expressed as

$$
H_{S_{1} S_{2} S_{3}}(\mathbf{s}) \equiv \sum_{i=0}^{L-1} S_{1}(i) S_{2}\left(i+s_{2}\right) S_{3}\left(i+s_{3}\right)
$$

where $\mathbf{s}=\left(s_{2}, s_{3}\right)$ represents the relative time shifts of $S_{2}$ and $S_{3}$, with reference to $S_{1}$.

Denote the intersection of sequences $\left\{S_{1}(i)\right\}$ and $\left\{S_{2}(i+\right.$ $\left.\left.s_{2}\right)\right\}$ by $\left\{S_{1} \cap S_{2}\left(i, s_{2}\right)\right\}$. If the number of coincidences between two sequences is shift invariant, by (39)

$$
\sum_{i=0}^{L-1} S_{1} \cap S_{2}\left(i, s_{2}\right)=\frac{L}{p^{2}}
$$

Similarly to (38), by (40) and (41)

$$
\begin{aligned}
\sum_{s_{3}=0}^{L-1} H_{S_{1} S_{2} S_{3}}(\mathbf{s}) & \equiv \sum_{s_{3}=0}^{L-1} \sum_{i=0}^{L-1} S_{1}(i) S_{2}\left(i+s_{2}\right) S_{3}\left(i+s_{3}\right) \\
& =\sum_{s_{3}=0}^{L-1} \sum_{i=0}^{L-1}\left(S_{1} \cap S_{2}\left(i, s_{2}\right)\right) S_{3}\left(i+s_{3}\right) \\
& =\frac{L}{p^{2}} \times \frac{L}{p} .
\end{aligned}
$$

Thus, if the number of common coincidences of the three sequences is also shift invariant, by the result of (42)

$$
H_{S_{1} S_{2} S_{3}}(\mathbf{s})=\frac{\left(L / p^{2}\right) \times(L / p)}{L}=\frac{L}{p^{3}}
$$

i.e., the three sequences commonly meet $L / p^{3}$ times.

If the number of common coincidences of $n$ sequences is shift invariant for $n=2,3, \ldots, k$, where $k \leq p$, i.e., $H_{S_{1}, S_{2}}, H_{S_{1}, S_{2}, S_{3}}, \ldots$, and $H_{S_{1}, S_{2}, S_{3}, \ldots, S_{k}}$ are shift invariant, by induction

$$
H_{S_{1} S_{2} S_{3} \ldots S_{k}}(\mathbf{s})=\frac{\left(L / p^{k-1}\right) \times(L / p)}{L}=\frac{L}{p^{k}}
$$

i.e., $k$ sequences commonly meet $L / p^{k}$ times. Therefore, for $p$ sequences, if $\left\{H_{S_{1}, S_{2}}, H_{S_{1}, S_{2}, S_{3}}, \ldots, H_{S_{1}, S_{2}, S_{3}, \ldots, S_{p}}\right\}$ is shift invariant

$$
H_{S_{1} S_{2} S_{3} \cdots S_{p}}(\mathbf{s})=\frac{L}{p^{p}} .
$$

Since, by definition, the sequences have positive duty factors, they must meet at least once, i.e.,

$$
L / p^{p} \geq 1
$$

which implies that

$$
L \geq p^{p} .
$$

Hence, one can conclude that, for the symmetric rate case, the minimum necessary period length of the $\left\{H_{S_{1}, S_{2}}\right.$, 
$\left.H_{S_{1}, S_{2}, S_{3}}, \ldots, H_{S_{1}, S_{2}, S_{3}, \ldots, S_{p}}\right\}$ shift-invariant protocol sequences is equal to $p^{p}$.

Note that, if only a number of $k$ protocol sequences are needed, where $2 \leq k \leq p$, the minimum sequence period length required for the $\left\{H_{S_{1}, S_{2}}, H_{S_{1}, S_{2}, S_{3}}, \ldots, H_{S_{1}, S_{2}, S_{3}, \ldots, S_{k}}\right\}$ shift-invariant cross-correlation property is $p^{k}$ by the result of $L / p^{k} \geq 1$. In addition, this shift-invariant cross-correlation property also implies shift-invariant individual and system throughputs. Consequently, the individual throughput can be expressed as

$$
\sum_{i=1}^{k}(1 / p)^{i}(-1)^{i-1}\left(\begin{array}{c}
k-1 \\
i-1
\end{array}\right)
$$

whereas the system throughput is equal to

$$
k \sum_{i=1}^{k}(1 / p)^{i}(-1)^{i-1}\left(\begin{array}{c}
k-1 \\
i-1
\end{array}\right) .
$$

They are consistent with (32) and (33).

\section{ACKNOWLEDGMENT}

The authors would like to thank the anonymous reviewers for their valuable and detailed comments and K. W. Shum and C. W. Sung for their insightful comments and discussions.

\section{REFERENCES}

[1] S. Haykin, Communication Systems. New York: Wiley, 1994, pp. 579-586.

[2] P. Fan and M. Darnell, Sequence Design for Communications Applications. London, U.K.: Res. Stud. Press, 1996.

[3] I. F. Akyildiz, W. Su, Y. Sankarasubramaniam, and E. Cayirci, "A survey on sensor networks," IEEE Commun. Mag., vol. 40, no. 8, pp. 102-114, Aug. 2002.

[4] J. Hill, R. Szewczyk, A. Woo, S. Hollar, D. Culler, and K. Pister, "System architecture directions for networked sensors," ACM SIGPLAN Not., vol. 35 , no. 11, pp. 93-104, Nov. 2000.

[5] I. Demirkol, C. Ersoy, and F. Alagoz, "MAC protocols for wireless sensor networks: A survey," IEEE Commun. Mag., vol. 44, no. 4, pp. 115-121, Apr. 2006.

[6] R. Jurdak, C. V. Lopes, and P. Baldi, "A survey, classification and comparative analysis of medium access control protocols for ad hoc networks," Commun. Surveys Tuts., vol. 6, no. 1, pp. 2-16, Jan. 2004.

[7] J. L. Massey, "The capacity of the collision channel without feedback," in Proc. IEEE Int. Symp. Inf. Theory, Jun. 1982, p. 101.

[8] G. Thomas, "Capacity of the wireless packet collision channel without feedback," IEEE Trans. Inf. Theory, vol. 46, no. 3, pp. 1141-1144, May 2000.

[9] J. L. Massey and P. Mathys, "The collision channel without feedback," IEEE Trans. Inf. Theory, vol. IT-31, no. 2, pp. 192-204, Mar. 1985.

[10] E. L. Titlebaum, "Time-frequency HOP signals-Part I: Coding based upon the theory of linear congruences," IEEE Trans. Aerosp. Electron. Syst., vol. AES-17, no. 4, pp. 490-493, Jul. 1981.

[11] A. A. Shaar and P. A. Davies, "Prime sequences: Quasi-optimal sequences for OR channel code division multiplexing," Electron. Lett., vol. 19, no. 21, pp. 888-890, Oct. 1983.

[12] P. R. Prucnal, M. A. Santoro, and T. R. Fan, "Spread spectrum fiberoptic local area network using optical processing," J. Lightwave Technol., vol. LT-4, no. 5, pp. 547-554, May 1986.
[13] A. A. Shaar and P. A. Davies, "A survey of one-coincidence sequences for frequency-hopped spread-spectrum systems," Proc. Inst. Electr. Eng., vol. 131, no. 7, pt. F, pp. 719-724, Dec. 1984.

[14] A. A. Shaar, M. Gharib, and P. A. Davies, "Collision resolution in contention access local area networks using concatenated prime sequences," Proc. Inst. Electr. Eng.-Commun., vol. 149, no. 5, pp. 249-256, Oct. 2002.

[15] Q. A. Nguyen, L. Györfi, and J. L. Massey, "Constructions of binary constant-weight cyclic codes and cyclically permutable codes," IEEE Trans. Inf. Theory, vol. 38, no. 3, pp. 940-949, May 1992.

[16] L. Györfi and I. Vajda, "Constructions of protocol sequences for multiple access collision channel without feedback," IEEE Trans. Inf. Theory, vol. 39, no. 5, pp. 1762-1765, Sep. 1993.

[17] W. S. Wong, "New protocol sequences for random-access channels without feedback," IEEE Trans. Inf. Theory, vol. 53, no. 6, pp. 2060-2071, Jun. 2007.

[18] G.-C. Yang and W. C. Kong, Prime Codes With Applications to Optical and Wireless Networks. Boston, MA: Artech House, 2002.

[19] V. C. da Rocha, Jr., "Protocol sequences for collision channel without feedback," Electron. Lett., vol. 36, no. 24, pp. 2010-2012, Nov. 2000.

[20] S. Tinguely, M. Rezaeian, and A. J. Grant, "The collision channel with recovery," IEEE Trans. Inf. Theory, vol. 51, no. 10, pp. 3631-3638, Oct. 2005.

[21] R. G. Gallager, "A perspective on multiaccess channels," IEEE Trans. Inf. Theory, vol. IT-31, no. 2, pp. 124-142, Mar. 1985.

[22] J. Y. N. Hui, "Multiple accessing for the collision channel without feedback," IEEE J. Sel. Areas Commun., vol. SAC-2, no. 4, pp. 575-582, Jul. 1984.

[23] C. S. Chen and W. S. Wong, "Bandwidth allocation for wireless multimedia systems with most regular sequences," IEEE Trans. Wireless Commun., vol. 4, no. 2, pp. 635-645, Mar. 2005.

[24] L. G. Roberts, "Dynamic allocation of satellite capacity through packet reservations," in Proc. Nat. Comput. Conf., 1973, pp. 711-716.

[25] Wireless LAN Medium Access Control (MAC) and Physical Layer (PHY) Specifications, IEEE Std. 802.11, IEEE-SA Standards Board, 1999.

[26] Wireless Medium Access (MAC) and Physical Layer (PHY) Specifications for Low-Rate Wireless Personal Area Networks (WPANs), IEEE Std. 802.15.4-2006, IEEE-SA Standards Board, Sep. 2006.

[27] S. Csibi and L. Györfi, "Random time and frequency hopping for unslotted asynchronous access," in Proc. IEEE Int. Symp. Spread Spectrum Tech. Appl., Mainz, Germany, Sep. 1996, pp. 1123-1127.

[28] C. S. Chen, W. S. Wong, and Y. Q. Song, "The design and analysis of protocol sequences for robust wireless accessing," in Proc. IEEE GLOBECOM, Washington, DC, 2007, pp. 1-6.

[29] D. V. Sarwate and M. B. Pursley, "Crosscorrelation properties of pseudorandom and related sequences," Proc. IEEE, vol. 68, no. 5, pp. 593-619, May 1980.

[30] C. S. Chen and W. S. Wong, "A robust access protocol for wireless sensor networks," in Proc. IEEE Mil. Commun. Conf., Washington, DC, Oct. 2006, pp. 1-6.

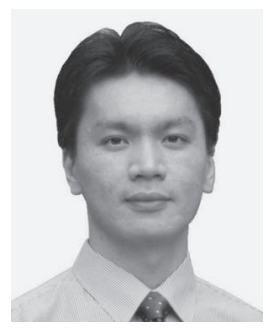

Chung Shue Chen (S'02-M'05) received the B.Eng., M.Phil., and Ph.D. degrees in information engineering from The Chinese University of Hong Kong, Shatin, Hong Kong, in 1999, 2001, and 2005 , respectively.

In 2002, he was with the National Institute for Telecommunications (INT), Evry, France, as a summer Intern. During 2005-2006, he was a Visiting Assistant Professor with The Chinese University of Hong Kong. During 2006-2007, he was a Postdoctoral Researcher with the Lorraine Laboratory of IT Research and Applications (LORIA)-National Center for Scientific Research (CNRS), Villers Les Nancy, France. He is currently a Postdoctoral Research Fellow with the Department of Electronics and Telecommunications, Norwegian University of Science and Technology, Trondheim, Norway. His research interests include radio resource allocation, multiple-access control, signal processing, and quality-of-service management for wireless communication systems.

Dr. Chen has served as Technical Program Committee member of several international conferences, including IEEE CCNC, Globecom, and WCNC. He was the recipient of the Sir Edward Youde Memorial Fellowship in 2003 and the ERCIM "Alain Bensoussan" Fellowship in 2007. 


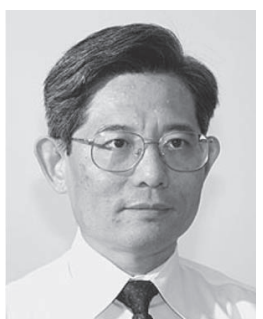

Wing Shing Wong (M'81-SM'90-F'02) received a combined B.A. and M.S. degree from Yale University, New Haven, CT, in 1976 and the M.S. and Ph.D. degrees from Harvard University, Cambridge, MA, in 1978 and 1980, respectively.

In 1982, he joined AT\&T Bell Laboratories, Holmdel, NJ. From 1987 to 1992, he managed a group of technical staff working on a number of research and development projects and consulting activities. In 1992, he joined The Chinese University of Hong Kong, Shatin, Hong Kong, where he is currently a Professor of information engineering with the Department of Information Engineering and the Dean of the Graduate School. From 1995 to 2003, he was the Chairman of the Department of Information Engineering. He is also a co-Editor-in-Chief of the Communications in Information and Systems. He is actively involved in a variety of research-and-development projects, including topics such as mobile communication systems, search engine development, and joint control-communication problems.

Dr. Wong was an Associate Editor of the IEEE TRANSACTIONS ON Automatic Control for four years. From 2003 to 2005, he served as Science Advisor at the Innovation and Technology Commission of the HKSAR government and is currently a Board Director and Chairman of the Technical Committee of the Hong Kong Applied Science and Technology Research Institute.

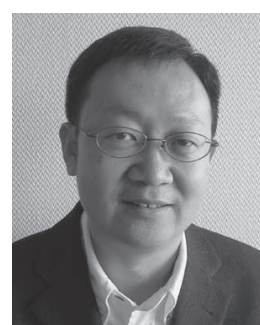

science.

In 1988, he joined Lorraine Laboratory of IT Research and Applications (LORIA)-INPL, Vandoeuvre-les-Nancy, France. From 1992 to 2005, he was an Assistant and then Associate Professor with the University of Henri Poincare Nancy 1. From 2001 to 2003, he was a Full-Time Researcher with INRIA Lorraine. He is currently a Professor of computer science with the LORIA-INPL. His research interests include modeling and performance evaluation of networks and real-time distributed systems by queueing analysis, network calculus and scheduling theory, and the implementation of real-time quality-of-service mechanisms in industrial networks, in-vehicle networks, IP networks, power line communication networks, and wireless sensor networks. 